\title{
Lifetime study of particle-hole excitations in the semimagic nucleus ${ }^{93} \mathrm{Tc}$
}

\author{
M. Hausmann, ${ }^{1}$ A. Jungclaus, ${ }^{2,3}$ E. Galindo, ${ }^{1}$ K. P. Lieb, ${ }^{1}$ O. Yordanov, ${ }^{1}$ I. P. Johnstone, ${ }^{4}$ R. Schwengner, ${ }^{5}$ A. Dewald, ${ }^{6}$ \\ A. Fitzler, ${ }^{6}$ O. Möller, ${ }^{6}$ G. de Angelis, ${ }^{7}$ A. Gadea, ${ }^{7}$ T. Martinez, ${ }^{7}$ D. R. Napoli, ${ }^{7}$ and C. $\mathrm{Ur}^{7}$ \\ ${ }^{1}$ II. Physikalisches Institut, Universität Göttingen, D-37073 Göttingen, Germany \\ ${ }^{2}$ Departamento de Física Teórica C-XI, Universidad Autónoma de Madrid, E-28049 Madrid, Spain \\ ${ }^{3}$ Instituto de Estructura de la Materia, Consejo Superior de Investigaciones Científicas, E-28006 Madrid, Spain \\ ${ }^{4}$ Department of Physics, Queen's University, Kingston, Ontario, Canada K7L 3N6 \\ ${ }^{5}$ Institut für Kern- und Hadronenphysik, FZ Rossendorf, D-01314 Dresden, Germany \\ ${ }^{6}$ Institut für Kernphysik, Universität zu Köln, D-50937 Köln, Germany \\ ${ }^{7}$ Istituto Nazionale di Fisica Nucleare, Laboratori Nazionali di Legnaro, I-35020 Legnaro, Italy
}

(Received 28 March 2003; published 18 August 2003)

\begin{abstract}
The recoil-distance Doppler-shift technique was employed for determining lifetimes of high-spin states in the semimagic nucleus ${ }^{93} \mathrm{Tc}$. The nuclei were populated using the reaction ${ }^{64} \mathrm{Zn}\left({ }^{35} \mathrm{Cl}, \alpha 2 p\right)$ at a beam energy of $135 \mathrm{MeV}$, and the $\gamma$ radiation from their decay was detected in the GASP spectrometer. A total of 26 reduced transition probabilities and limits for 19 further transitions were extracted and compared to large-scale shell model calculations, considering different configuration spaces and residual interactions. The information deduced about transition strengths turned out to be essential for the correct assignment of the calculated to the experimental excited states.
\end{abstract}

DOI: 10.1103/PhysRevC.68.024309

PACS number(s): 21.10.Tg, 21.60.Cs, 23.20.Js, 27.60.+j

\section{INTRODUCTION}

In the present paper, we extend our study of the interplay between neutron core excitations and $f_{5 / 2}, p_{3 / 2} \rightarrow g_{9 / 2}$ proton excitations in the neutron deficient $N=50$ isotones at intermediate spin to the nucleus ${ }^{93} \mathrm{Tc}$. Our earlier lifetime studies on ${ }^{94} \mathrm{Ru}$ and ${ }^{95} \mathrm{Rh}[1,2]$ had demonstrated that only on the basis of experimental electromagnetic transition strengths the contributions of the different excitation modes can be disentangled. The level scheme of ${ }^{93} \mathrm{Tc}$ at high spin has been reported in three papers. Grecescu et al. [3] used the ${ }^{92} \operatorname{Mo}(\alpha, p 2 n)$ reaction to establish the level sequences up to spin 25/2. After this first in-beam study the level scheme has been considerably extended by Ghugre et al. [4] and Roth et al. [5], both employing heavy-ion-induced fusion-evaporation reactions. The much higher efficiency of the detector arrangements employed in these recent experiments enabled the observation of many new states up to spins $43 / 2^{+}$and $43 / 2^{-}$, respectively. There are some severe discrepancies between the two published level schemes. Our analysis of electromagnetic transition strengths is based on the excitation scheme obtained by Roth et al. [5] which is shown in Fig. 1. States up to spin $21 / 2^{+}$and $25 / 2^{-}$can be built considering only the $\pi\left(g_{9 / 2}\right)^{3}$ and $\pi\left(p_{1 / 2}\right) \otimes \pi\left(g_{9 / 2}\right)^{4}$ configurations, respectively, and by lifting the $p_{1 / 2}$ proton pair from $p_{1 / 2}$ to $g_{9 / 2}$, a $25 / 2^{+}$can be constructed within the $\left(g_{9 / 2}, p_{1 / 2}\right)$ space, too. For higher-spin states, however, the breaking of the $N=50$ neutron core is required and indeed shell model calculations allowing for such a neutron excitation across the shell gap were very successful in describing the observed level schemes of some $N=50$ isotones [6]. Recently, however, we were able to prove that some states at intermediate spin in ${ }^{94} \mathrm{Ru}$ and ${ }^{95} \mathrm{Rh}$ are of pure proton structure including the excitation of a proton from the completely filled lower lying $f_{5 / 2}$ to the $g_{9 / 2}$ orbit $[1,2]$. Since this type of information can only be obtained on the basis of experimental electromagnetic transition rates, we now perform a coincidence lifetime measurement employing the recoil distance Doppler shift (RDDS) technique and the GASP spectrometer to determine accurate lifetimes, in particular, for high-spin states, in the neighboring isotone ${ }^{93} \mathrm{Tc}$.

The paper is organized as follows. In Sec. II, details about the lifetime experiment performed in the present study are given followed by a description of the data treatment in Sec. III. In Sec. IV, the determination of level lifetimes from the data using two different methods is illustrated. A new way of applying the differential decay curve method (DDCM) is introduced and the results obtained with this method are compared with the values deduced employing the standard DDCM as well as the conventional analyses of the decay curves. The resulting experimental transition strengths are compared to shell model calculations within different configuration spaces in Sec. V and in Sec. VI analogies between the $N=50$ isotones ${ }^{93} \mathrm{Tc}$, ${ }^{94} \mathrm{Ru}$, and ${ }^{95} \mathrm{Rh}$ are discussed. Finally, the paper is closed with some conclusions in Sec. VII.

\section{EXPERIMENT}

The reaction ${ }^{64} \mathrm{Zn}\left({ }^{35} \mathrm{Cl}, \alpha 2 p\right)$ was used to populate excited states in ${ }^{93} \mathrm{Tc}$ using $\mathrm{a} \approx 1$-pnA $135-\mathrm{MeV}{ }^{35} \mathrm{Cl}$ beam provided by the XTU tandem accelerator at the LNL Legnaro. A $1.0-\mathrm{mg} / \mathrm{cm}^{2}$ stretched ${ }^{64} \mathrm{Zn}$ target foil (enrichment 99.8\%) and a 7- $\mu \mathrm{m}$-stretched Au stopper foil were mounted in the Cologne plunger device [7] and data were taken at 13 different flight distances in the range from 20 to $6117 \mu \mathrm{m}$. The closest average distance, before the two foils got into electrical contact, was 17(5) $\mu \mathrm{m}$ and the distances were corrected accordingly. At a beam energy of $135 \mathrm{MeV},{ }^{93} \mathrm{Tc}$ is produced as the fourth or the fifth strongest reaction channel. The lifetime analysis of excited states in the $3 p n$ channel 


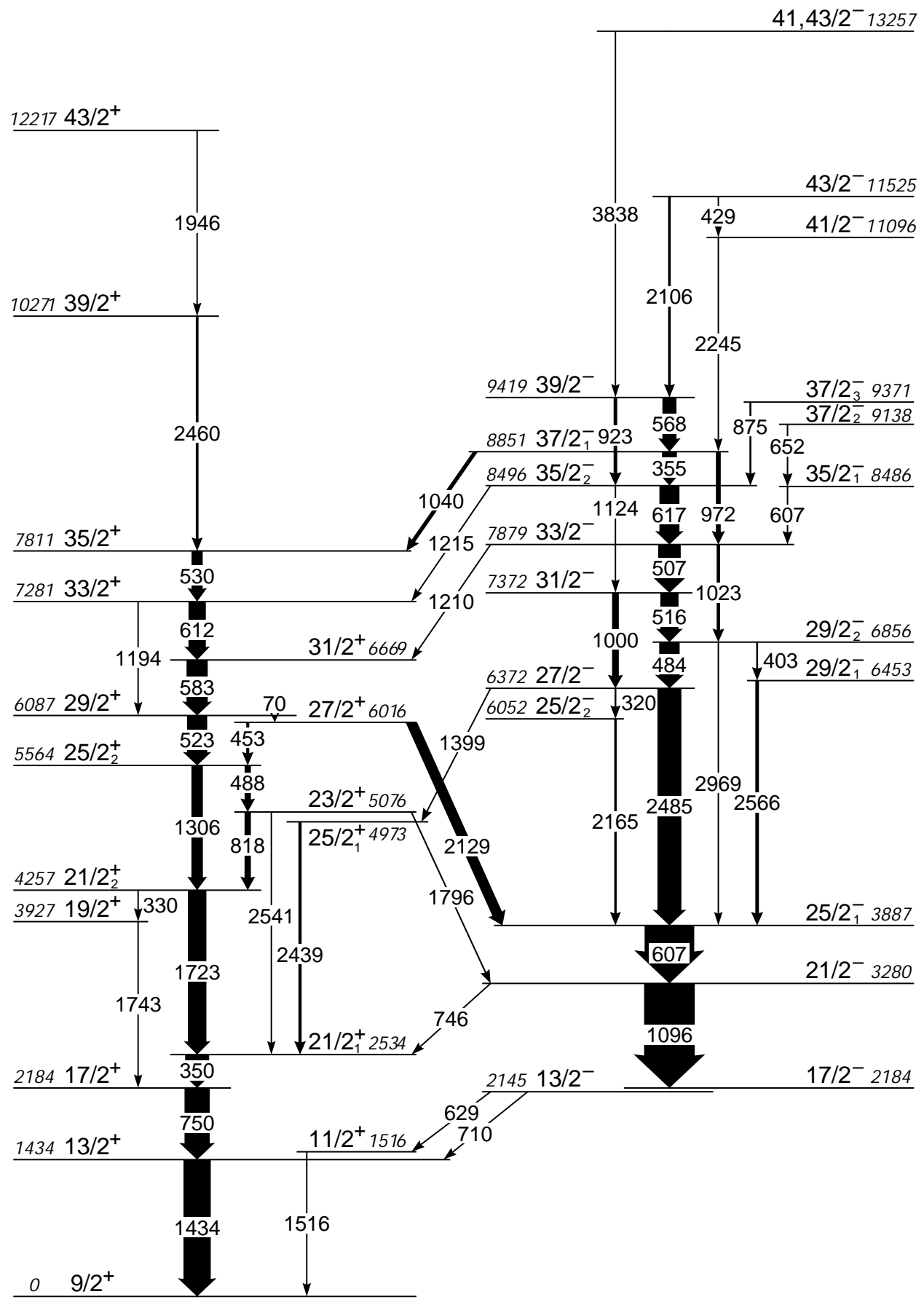

FIG. 1. Relevant part of the level scheme of ${ }^{93} \mathrm{Tc}$ deduced in Ref. [5]. Only the strongest transitions are included.

\footnotetext{
${ }^{95} \mathrm{Ru}$, the strongest populated in the same experiment, will be published elsewhere [8].

The $\gamma$ rays were detected in the GASP spectrometer [9] in close geometry with a photopeak efficiency of $3 \%$. The 40 Ge detectors of this $4 \pi$ spectrometer are arranged in seven rings at angles of $35^{\circ}, 59^{\circ}, 72^{\circ}, 90^{\circ}, 108^{\circ}, 121^{\circ}$, and $145^{\circ}$ with respect to the beam. The recoil velocity of the ${ }^{93} \mathrm{Tc}$ nuclei leaving the target foil was deduced from the energy shift between stopped and Doppler-shifted components of intense $\gamma$ transitions in ${ }^{93} \mathrm{Tc}$ to be $v=7.64(3) \mu \mathrm{m} / \mathrm{ps}$ or $2.55(1) \%$ of the speed of light.
}

\section{DATA TREATMENT}

The first step in the analysis has been a proper energy calibration using standard sources and a correction for time dependent energy shifts for the individual Ge detectors. Then, a symmetric $\gamma \gamma$ matrix was sorted containing coincidences between all 40 detectors of GASP for the shortest flight distance of $20 \mu \mathrm{m}$. This matrix was used to check the known level scheme of ${ }^{93} \mathrm{Tc}$ [5]. Since no discrepancies were observed, the following lifetime analysis was based on the level scheme of Roth et al. [5], which is shown in Fig. 1. For the lifetime analysis, for each of the 13 target-to-stopper dis- 


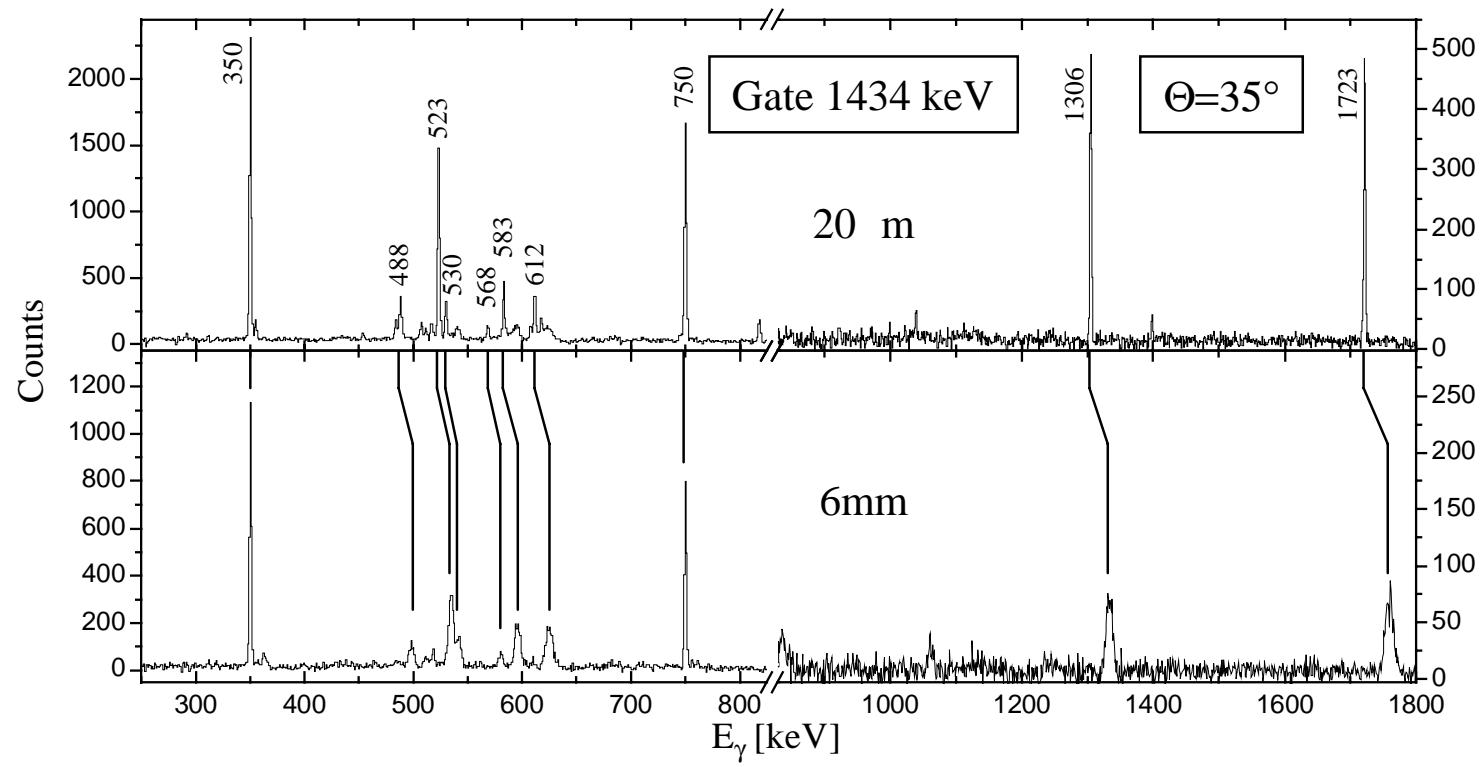

FIG. 2. Coincidence spectra observed at $35^{\circ}$ with gate set on the 1434-keV ground state transition at flight distances of $20 \mu \mathrm{m}$ and $6 \mathrm{~mm}$.

tances, the $\gamma \gamma \gamma$ coincidences and higher-fold events were unfolded to twofold events and sorted into asymmetric matrices, with one particular ring of GASP on each axis. Considering the seven rings at different angles with respect to the beam, there are 28 possible combinations for $\gamma \gamma$ coincidences. Altogether, 364 matrices were sorted for the complete dataset. The matrices containing data from identical rings on both axes were symmetrized in order to achieve full counting statistics in each gate on one axis. For each pair of coincident $\gamma$ rays of interest, a set of 49 independent coincidence spectra was obtained from these matrices corresponding to the 49 possible combinations of detecting the "gate" and the "look" transition at one of the seven different angles. After a careful check for contaminations, which might occur only for certain detection angles due to the angular dependence of the position of the Doppler-shifted lines, the 49 decay curves determined from these coincidence spectra can be added up.

Gates were first set on the unshifted components of intense low-lying $\gamma$ transitions observed in all detectors. As an example, the spectrum measured at $35^{\circ}$ for flight distances of $20 \mu \mathrm{m}$ and $6 \mathrm{~mm}$ in coincidence with the 1434-keV ground state transition is shown in Fig. 2. As can be seen in this figure, only two transitions of 750 and $350 \mathrm{keV}$ below the known $21 / 2^{+}$isomer do not show any significant Dopplershifted component even for the long flight distance of $6 \mathrm{~mm}$. For all the other transitions, the unshifted line observed in the spectrum taken at $20 \mu \mathrm{m}$ is shifted to higher energies in the spectrum measured at the long flight distance. In Fig. 3, coincidence spectra with gate on the $1096-\mathrm{keV} 21 / 2^{-} \rightarrow$ $17 / 2^{-}$transition are shown for two different flight distances to illustrate the situation for the negative parity transitions. Here, only the $607-\mathrm{keV} 25 / 2^{-} \rightarrow 21 / 2^{-}$transition still shows a large unshifted component in the spectrum taken at $200 \mu \mathrm{m}$. To get a first qualitative impression of the time structure of the $\gamma$ flux through the nucleus, decay curves for all strong transitions within the main cascades at both pari- ties were deduced from these spectra. These "effective" decay curves are shown in Fig. 4. The intensities $R(x)$ in the coincidence spectra taken at the 13 target-to-stopper distances were normalized to the sum of the unshifted and shifted components of some strong transitions in ${ }^{93} \mathrm{Tc}$ in coincidence spectra with gates set on both unshifted and shifted parts of intense low-lying $\gamma$ rays.

\section{LIFETIME ANALYSIS AND RESULTS}

Since the counting statistics obtained for the strong reaction channels in the present experiment was high, it was possible to apply the DDCM [10] in the lifetime analysis, thus avoiding systematical uncertainties concerning cascade and side feedings. In this method the lifetime of a state is deduced for each target-to-stopper distance $x$ from the coincidence intensities of $\gamma$-rays feeding and depopulating the level of interest. If $\gamma_{i n}$ denotes a direct feeding transition and $\gamma_{\text {out }}$ a $\gamma$-ray depopulating the state, the lifetime can be calculated from the equation

$$
\tau(x)=\frac{1}{v} \frac{I\left(\gamma_{\text {out }}^{\text {unshifted }}, \gamma_{\text {in }}^{\text {shifted }} ; x\right)}{d I\left(\gamma_{\text {out }}^{\text {shifted }}, \gamma_{\text {in }}^{\text {shifted }} ; x\right) / d x},
$$

where $I\left(\gamma_{\text {out }}^{\text {unshifted }}, \gamma_{\text {in }}^{\text {shifted }} ; x\right)$ is the intensity of the unshifted (stop) component of transition $\gamma_{\text {out }}$ in the coincidence spectrum with the gate set on the Doppler-shifted (flight) component of transition $\gamma_{i n}$. The denominator of Eq. 1 is derived from a fit of a smooth curve to the discrete values of $I\left(\gamma_{\text {out }}^{\text {shifted }}, \gamma_{\text {in }}^{\text {shifted }} ; x\right)$ for the different distances $x$. The lifetime is finally calculated by averaging the $\tau$ values obtained for the distances within the region of highest sensitivity, i.e., the range with largest values for both the numerator and the denominator of Eq. (1). As an example, the DDCM analysis of the lifetime of the $27 / 2^{-}$state at $6372 \mathrm{keV}$ is shown in Fig. 5. The gate was set on the $484-\mathrm{keV}$ feeding transition 


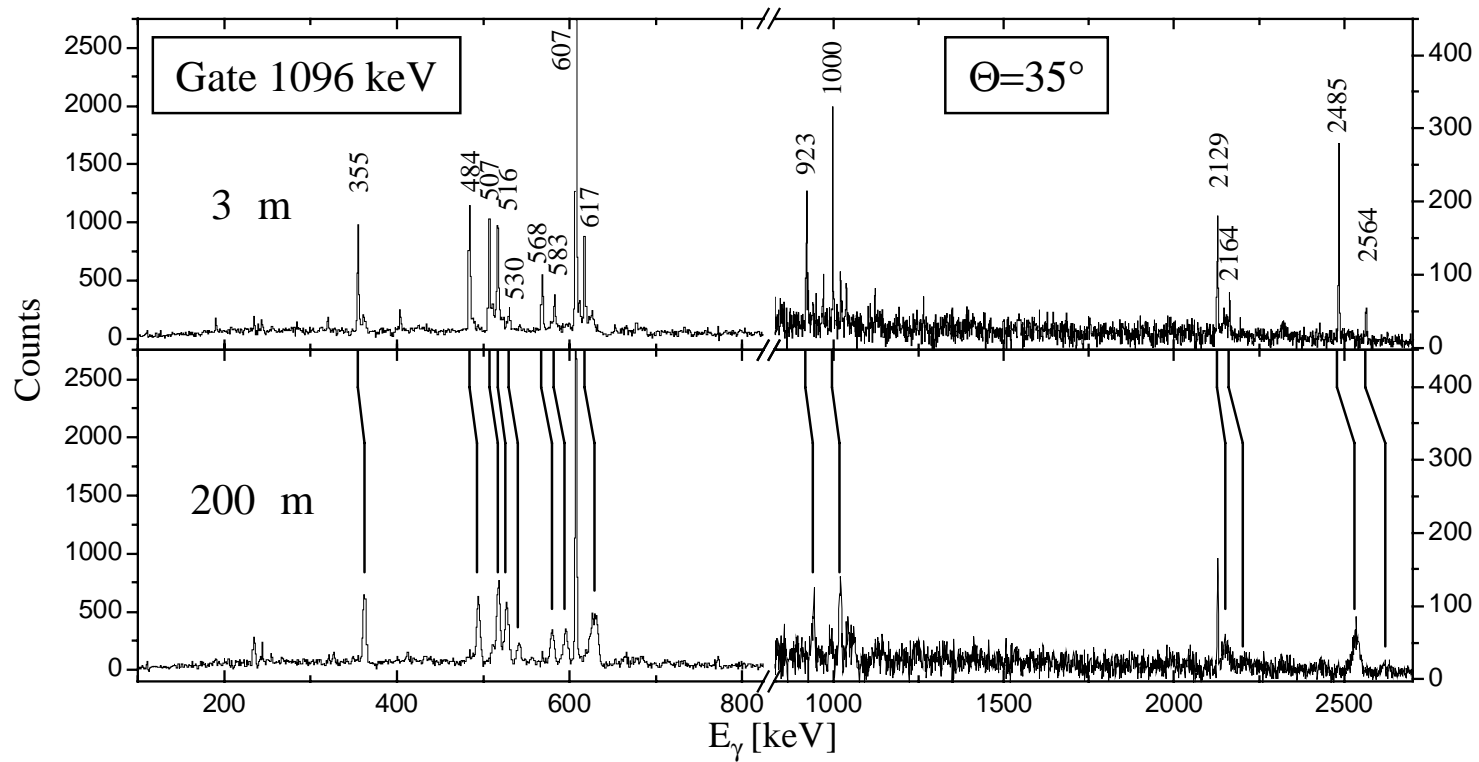

FIG. 3. Coincidence spectra observed at $35^{\circ}$ with gate set on the $1096-\mathrm{keV} 21 / 2^{-} \rightarrow 17 / 2^{-}$transition at flight distances of 3 and $200 \mu \mathrm{m}$.

and in this coincidence spectrum the intensities of the unshifted and Doppler-shifted components of the depopulating 2485-keV transition were determined [see Figs. 5(a) and 5(e)]. The slope of the shifted intensity, i.e., the denominator of Eq. (1), is shown in Fig. 5(b) and the resulting lifetimes are given in Fig. 5(c). The region of highest sensitivity, i.e., largest values for both the numerator and the denominator of Eq. (1), is marked by dashed vertical lines and averaging the lifetimes values in this region leads to $\tau\left(27 / 2^{-}\right)$ $=0.30(3)$ ps.

In some cases, however, the determination of the slope of the shifted intensity is not unequivocal. Since the functional dependence of the flight peak intensity on the distance is unknown, any approach leading to a reasonably smooth fit of the experimental values is allowed. Equivalent to Eq. (1), instead of dividing the stop peak intensity by the derivative of the flight peak intensity, one might as well go the integral form of this equation:

$$
\tau(x)=\frac{1}{v} \frac{\int_{x_{0}}^{x} I\left(\gamma_{\text {out }}^{\text {unshifted }}, \gamma_{\text {in }}^{\text {shifted }} ; x\right) d x}{I\left(\gamma_{\text {out }}^{\text {shifted }}, \gamma_{\text {in }}^{\text {shifted }} ; x\right)-I\left(\gamma_{\text {out }}^{\text {shifted }}, \gamma_{\text {in }}^{\text {shifted }} ; x_{0}\right)} .
$$

The evaluation of the same data as in the conventional DDCM analysis discussed above, but now using Eq. (2) in-
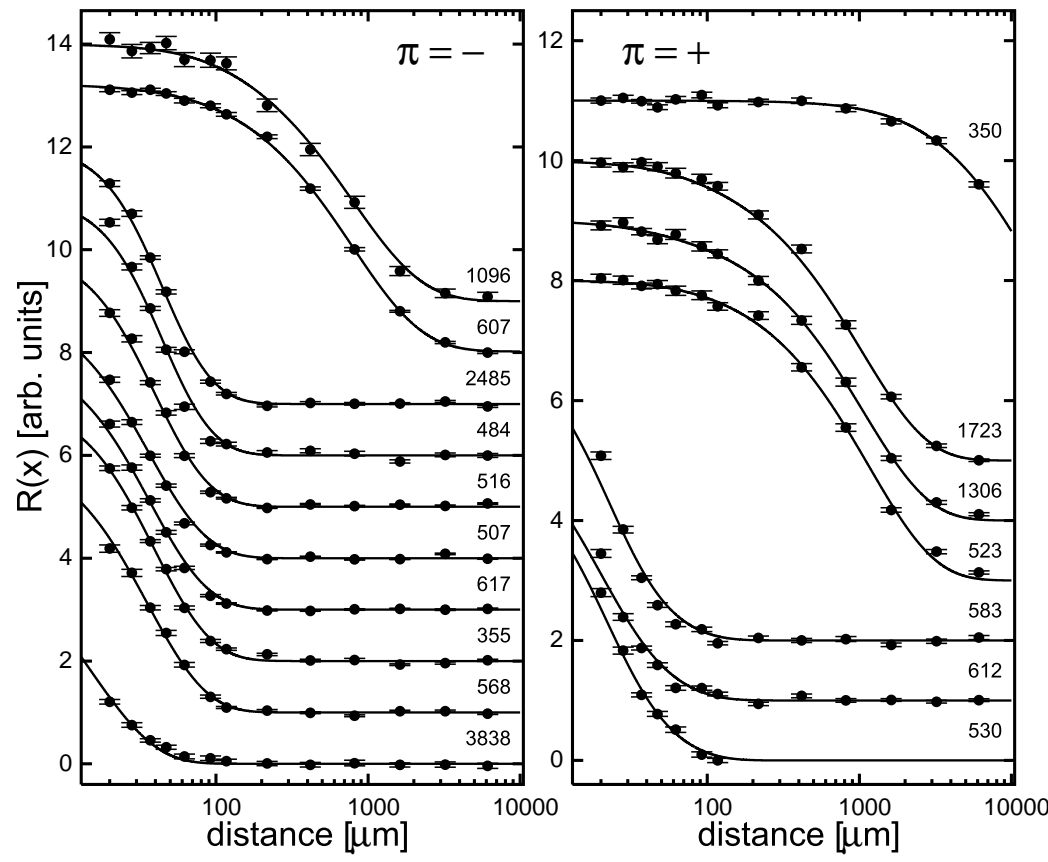

FIG. 4. "Effective" decay curves $R(x)$ for strong transitions determined in the coincidence spectra with gates set on the 1434$\mathrm{keV}$ line for the positive parity and the 1096-keV line for the negative parity. 


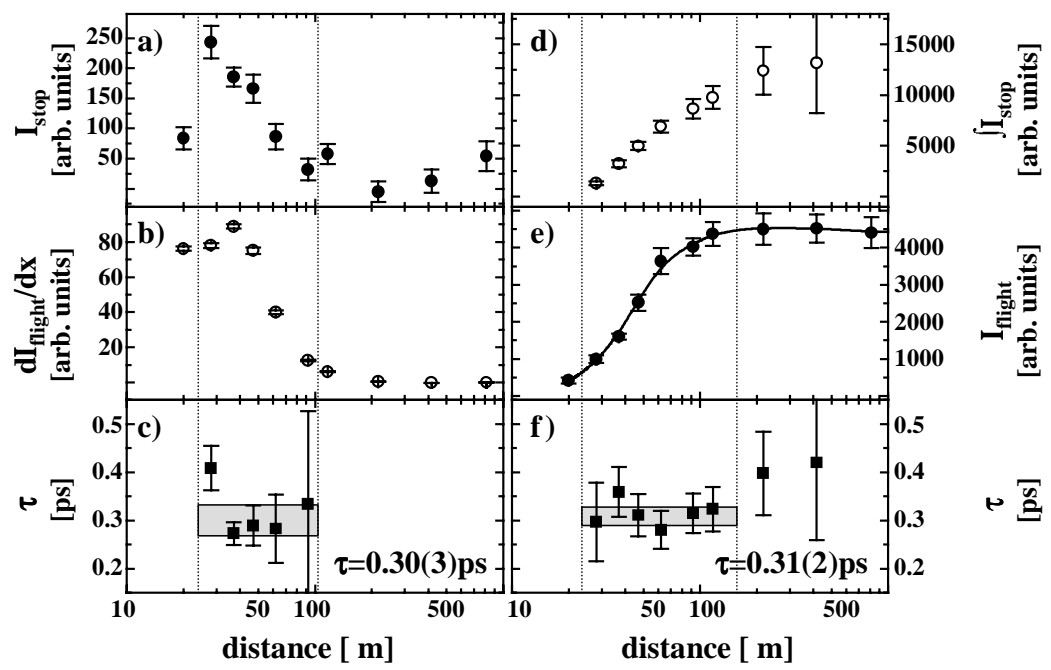

FIG. 5. Comparison between the DDCM analyses using Eqs. (1) and (2) for the $27 / 2^{-}$state at $6372 \mathrm{keV}$ in ${ }^{93}$ Tc. The experimental information, namely, the intensities of the unshifted and the Doppler-shifted intensities of the 2485-keV line in the coincidence spectrum with gate set on the shifted part of the 484-keV feeding transition is shown in parts (a) and (e). The derivative of the flight peak [denominator of Eq. (1)] is plotted in (b) and the integral of the unshifted intensity [numerator of Eq. (2) in (d)]. In the bottom row, the resulting lifetimes $\tau$ are shown for (c) using Eq. (1) and (f) using Eq. (2). See text for more details.

stead of Eq. (1), is illustrated in Figs. 5(d)-5(f). In Fig. 5(d), the integral stop peak intensity is plotted versus the distance and part (e) of this figure shows the flight peak intensity. Finally, the lifetime calculated using Eq. (2) is shown in Fig. $5(\mathrm{f})$. The resulting average lifetime value is $\tau\left(27 / 2^{-}\right)$ $=0.31(2) \mathrm{ps}$ in perfect agreement with the result obtained using Eq. (1).

The lifetime analysis in ${ }^{93} \mathrm{Tc}$ had to be done very carefully since many of the strong transitions have energies in the same range $(484,507,516,523,530,568,583,607,612$, $617 \mathrm{keV}$, etc.) leading in many cases to contaminations of either the shifted or unshifted components of the lines of interest. The spectra obtained with detectors with different angles with respect to the beam were individually checked and only the angle combinations without any contamination were considered. In the positive parity sequence, lifetimes of the $31 / 2^{+}, 29 / 2^{+}, 25 / 2_{2}^{+}$, and $21 / 2^{+}$levels could be determined using the DDCM method. Only for the $33 / 2^{+}$level, we did not succeed since it has not been possible to clearly separate the unshifted and shifted parts of the depopulating $612-\mathrm{keV} \gamma$ ray from the contaminations by the $607-\mathrm{keV}$ $25 / 2^{-} \rightarrow 21 / 2^{-}$transition in coincidence with the feeding $530-\mathrm{keV}$ transition. Within the main sequence at negative parity, the lifetime of the $33 / 2^{-}$state could not be determined due to problems to set proper gates on the flight component of the $617-\mathrm{keV}$ line, since this peak is contaminated by the $607-\mathrm{keV}$ transition. For the $21 / 2^{-}$level, the lifetime is so short that no unshifted component of the 1096-keV $\gamma$ ray was observed in coincidence with the Doppler-shifted part of the 607-keV transition at any measured flight distance. As examples of the DDCM analysis, determination of the lifetimes of the $25 / 2^{-}[\tau=86(8) \mathrm{ps}]$, the $31 / 2^{-}[\tau$ $=1.29(11) \mathrm{ps}]$, and the $37 / 2^{-}[\tau=1.02(8) \mathrm{ps}]$ states is illustrated in Fig. 6.

In the next step, we will study whether additional lifetime information can be deduced from the decay curves shown in Fig. 4. From this figure it is obvious that the analysis of these decay curves will be sensitive mainly to the lifetimes of the $35 / 2^{+}$(decay curve of the $530 \mathrm{keV}$ line), 29/2 ${ }^{+}$(523 keV), and $21 / 2^{+}(350 \mathrm{keV})$ states at positive parity and the $39 / 2^{-}$ $(568 \mathrm{keV})$ and $25 / 2^{-}(607 \mathrm{keV})$ levels at negative parity. For the $\gamma$ rays connecting these states, no significant changes in the decay curves are observed indicating short lifetimes of all the intermediate states. In the fit of the decay curves mentioned above, the complete known feeding pattern above the state under consideration was taken into account in all cases. For the $29 / 2^{+}$and the $25 / 2^{-}$states, the lifetimes obtained from such a fit of the decay curves can be compared to the results of the DDCM analysis. In both cases, the values deduced using these two independent methods are in excellent agreement: $153(10)$ vs $145(14)$ ps for the $29 / 2^{+}$state and $83(3)$ vs $86(8)$ ps for the $25 / 2^{-}$level. For the isomeric $21 / 2^{+}$ state, a lifetime of $\tau\left(21 / 2^{+}\right)=2.083(135)$ ns has been obtained from a fit of the decay curve of the $350-\mathrm{keV}$ transition. This value is in agreement with the literature value of 2.32(14) ns from Ref. [11] and slightly shorter than the value of 2.63(14) ns obtained in Ref. [12]. Finally, for the highestspin states, strongly populated in this experiment, namely, the $35 / 2^{+}$and $39 / 2^{-}$levels, lifetimes of $0.90(16)$ and 3.8(1.3) ps, respectively, have been determined. As mentioned above, the similarity of many of the decay curves in Fig. 4 indicates short intermediate lifetimes. Whereas it is difficult to determine the lifetime values from a fit of these curves, the upper lifetimes limits can be obtained taking into account the uncertainties of all parameters of the feeding scenario which enters into the fit.

All the lifetime results obtained in this work using the two different methods, namely DDCM and RDDS, are summarized in Table I. In Table II, the reduced electromagnetic transition probabilities derived from the experimental results 

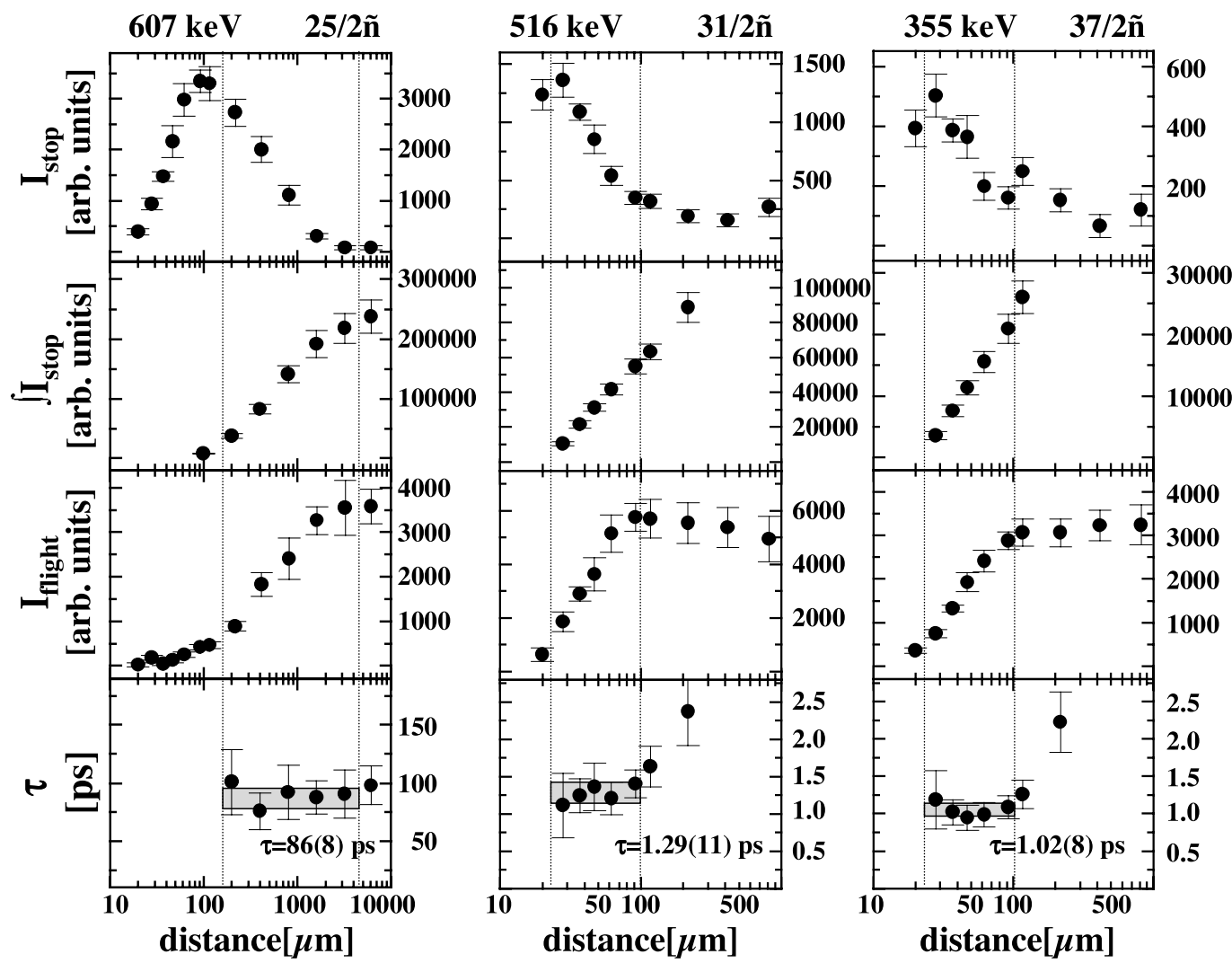

FIG. 6. DDCM analysis of the data for the $25 / 2^{-}, 31 / 2^{-}$, and $37 / 2^{-}$levels referring to the 607,516 , and $355 \mathrm{keV}$ transitions. The lifetimes obtained are shown in the lower parts where the $\tau$ curves are displayed and the average values with their errors are indicated by gray boxes. The region of highest sensitivity is marked by vertical dashed lines. For more details, see text.

are presented, making use of the branching ratios published in Ref. [5]. For the $\Delta I=0$ and $\Delta I=1$ transitions, the unknown mixing ratios were set to zero. In view of the mainly strong $M 1$ and weak $E 2$ transitions in this nucleus, this assumption seems well justified.

\section{COMPARISON TO SHELL MODEL CALCULATIONS}

To describe the level scheme of ${ }^{93} \mathrm{Tc}$ up to spins as high as $43 / 2^{+}$and $43 / 2^{-}$, neutron excitations across the $N=50$ shell gap have to be considered. On the proton side, excitations from the $f_{5 / 2}$ and $p_{3 / 2}$ orbits into the $g_{9 / 2}$ shell might also be of importance. To facilitate the discussion, we will classify the possible configurations in the following way: states built from protons within the $\left(p_{1 / 2}, g_{9 / 2}\right)$ space with an inert neutron core will be called core states. States containing a neutron $d_{5 / 2}$ particle-hole excitation will be referred to as $\nu p h$ and those which are dominated by proton $f_{5 / 2}, p_{3 / 2}$ $\rightarrow g_{9 / 2}$ excitations as $\pi p h$ states. Finally, levels with significant contributions from both neutron and proton excitations will be called $\pi \nu p h$. In the following we will discuss shell model (SM) calculations performed in two different configuration spaces. In the first (SM1), only neutron core excitations will be taken into account, neglecting possible excitations from the $f_{5 / 2}$ and $p_{3 / 2}$ proton states. Then, in a next step, we will try to describe both types of excitations simultaneously (SM2, SM3).

\section{A. Shell model calculation by Johnstone and Skouras (SM1)}

Recently, Johnstone and Skouras [6] performed shell model calculations using a ${ }^{88} \mathrm{Sr}$ core and allowing singleparticle excitations from the $p_{1 / 2}$ and $g_{9 / 2}$ orbits into the $d_{5 / 2}$, $s_{1 / 2}, d_{3 / 2}, g_{7 / 2}$ orbits for both protons and neutrons, neglecting however the $f_{5 / 2}$ and $p_{3 / 2}$ proton orbits (no $\pi p h$ states). The interaction used was determined largely by fits to levels of $N=49$ and $N=50$ nuclei; for details we refer to Ref. [6]. First of all, in this calculation the $s_{1 / 2}, d_{3 / 2}$, and $g_{7 / 2}$ orbits do not contribute significantly up to spins of $39 / 2^{+}$and $39 / 2^{-}$. The only exception at positive parity is the $37 / 2^{+}$ yrast level which in the calculation has a $g_{7 / 2}$ neutron particle-hole structure as it is the case for the calculated $37 / 2_{3}^{-}$state at negative parity. Since $39 / 2^{-}$is the maximum spin which can be obtained with five protons in the $\left(p_{1 / 2}\right.$, $\left.g_{9 / 2}\right)$ space coupled to a $g_{9 / 2} \rightarrow d_{5 / 2}$ neutron excitation, the calculated configuration of the $41 / 2^{-}$state is the same except for the neutron being excited to the $g_{7 / 2}$ rather than the $d_{5 / 2}$ orbit.

Whereas all states up to the yrast $21 / 2^{+}$and $25 / 2^{-}$states are pure core states, most of the high-spin states above the observed energy gaps, i.e., above about 4 and $6 \mathrm{MeV}$ at positive and negative parities, respectively, were found to be almost pure $\nu p h$ states, with the neutron being excited to the $d_{5 / 2}$ shell. The only exceptions are the calculated $21 / 2_{2}^{+}$state at $4 \mathrm{MeV}$ and the yrast $25 / 2^{+}$level at $5 \mathrm{MeV}$, which were 
TABLE I. Lifetimes of excited states in ${ }^{93} \mathrm{Tc}$ as obtained in this work. The energy and spin/parity of the state are given in the first and second columns, respectively. The third column indicates the energy of the depopulating $\gamma$ ray used in the lifetime determination. $\tau_{D D C M}$ and $\tau_{R D D S}$ (columns 4 and 5) are the state lifetimes determined via the DDCM and RDDS methods, respectively.

\begin{tabular}{|c|c|c|c|c|}
\hline$E_{x}(\mathrm{keV})$ & $I^{\pi}$ & $E_{\gamma}(\mathrm{keV})$ & $\tau_{D D C M}(\mathrm{ps})$ & $\tau_{R D D S}(\mathrm{ps})$ \\
\hline \multicolumn{5}{|c|}{ Positive parity } \\
\hline 2184 & $17 / 2^{+}$ & 750 & & $39(7)^{\mathrm{a}}$ \\
\hline 2534 & $21 / 2^{+}$ & 350 & & $2083(135)^{b}$ \\
\hline 4257 & $21 / 2_{2}^{+}$ & 1723 & $4.79(52)$ & \\
\hline 4973 & $25 / 2^{+}$ & 2439 & & $<1.3$ \\
\hline 5076 & $23 / 2^{+}$ & 818 & & $<80$ \\
\hline 5564 & $25 / 2_{2}^{+}$ & 1306 & $10.0(15)$ & \\
\hline 6016 & $27 / 2^{+}$ & 2129 & & $<90$ \\
\hline 6087 & $29 / 2^{+}$ & 523 & $145(14)$ & $153(10)$ \\
\hline 6669 & $31 / 2^{+}$ & 583 & $0.34(4)$ & $<0.8$ \\
\hline 7281 & $33 / 2^{+}$ & 612 & & $<0.6$ \\
\hline 7811 & $35 / 2^{+}$ & 530 & & $0.90(16)$ \\
\hline 10271 & $39 / 2^{+}$ & 2460 & & $<1.9$ \\
\hline \multicolumn{5}{|c|}{ Negative parity } \\
\hline 3280 & $21 / 2^{-}$ & 1096 & & $<2.0$ \\
\hline 3887 & $25 / 2^{-}$ & 607 & $86(8)$ & $83(3)$ \\
\hline 6052 & $25 / 2_{2}^{-}$ & 2165 & & $<0.8$ \\
\hline 6372 & $27 / 2^{-}$ & 2485 & $0.31(2)$ & $<1.0$ \\
\hline 6453 & $29 / 2^{-}$ & 2566 & & $<0.8$ \\
\hline 6856 & $29 / 2_{2}^{-}$ & 484 & $1.32(7)$ & $<1.7$ \\
\hline 7372 & $31 / 2^{-}$ & 516 & $1.29(11)$ & $<1.7$ \\
\hline 7879 & $33 / 2^{-}$ & 507 & & $<0.4$ \\
\hline 8496 & $35 / 2_{2}^{-}$ & 617 & $0.59(9)$ & $<1.0$ \\
\hline 8851 & $37 / 2^{-}$ & 355 & $1.02(8)$ & $<1.2$ \\
\hline 9419 & $39 / 2^{-}$ & 568 & & $3.8(1.3)$ \\
\hline 11525 & $43 / 2^{-}$ & 2106 & & $<4.0$ \\
\hline 13257 & $41 / 2^{-}, 43 / 2^{-}$ & 3838 & & $<2.0$ \\
\hline
\end{tabular}



${ }^{\mathrm{b}}$ Literature values 2.32(14) ns [11] and 2.63(14) ns [12].

assigned pure proton character. The calculated excitation energies are compared to the experimental ones in Fig. 7. While the overall agreement between experimental and calculated excitation energies is very good, assigning the first calculated state of each spin to the first experimentally observed one, the second calculated to the second observed, and so on, it is worse than the average for some states. Examples are the yrast $29 / 2^{-}$as well as the yrare $21 / 2_{2}^{+}, 35 / 2_{2}^{-}$ and $37 / 2_{2}^{-}$states. As mentioned earlier [6] this might be due the fact that for some levels, $p_{3 / 2}$ or $f_{5 / 2}$ proton hole configurations are important. The best way to clarify the structure of these states is to compare the electromagnetic transition strengths calculated from the wave functions with the experimental results. For the $B(M 1)$ values the effective singleparticle $g$ factors $g_{s}^{e f f}=0.7 g_{s}^{\text {bare }}$ were used, where $g_{s}^{\text {bare }}$ denote the bare-nucleon $g$ factors. As usual, effective charges of $e_{\pi}=1.72 e$ and $e_{\nu}=1.44 e$ for protons and neutrons, respectively, were used to calculate $E 2$ transition probabilities. Whereas the second calculated $21 / 2^{+}$state is a pure proton state, there is some evidence that the second observed level is a $\nu p h$ state. First, it is strongly populated from the $25 / 2_{2}^{+}$ $\nu p h$ state $\left[B(E 2)=14(2) e^{2} \mathrm{fm}^{4}\right]$ and not from the yrast $25 / 2^{+}$level, which is a $\pi p h$ state, and second its decay to the yrast $21 / 2^{+}$state is rather weak $[B(M 1)=2.2(2)$ $\left.\times 10^{-3} \mu_{N}^{2}\right]$ indicating that a $d_{5 / 2} \rightarrow g_{9 / 2}$ neutron transition is involved. We therefore assign the observed $21 / 2_{2}^{+}$state to the calculated $21 / 2_{3}^{+}$level. The second calculated $21 / 2^{+}$state is not observed because it presumably is fed only very weakly by the higher-lying $\nu p h$ states. The calculated yrast $25 / 2^{+}$ state is a core state and corresponds to the observed $25 / 2^{+}$ level at $4973 \mathrm{keV}$. This state is populated by none of the positive parity $\nu p h$ states but only via a weak decay branch from the $27 / 2^{-}$level. It also decays only to the yrast $21 / 2^{+}$ core state $\left[B(E 2)>7 e^{2} \mathrm{fm}^{4}\right]$ and not to the $21 / 2_{2}^{+} \nu p h$ level. At negative parity, the main flux goes through the M1 cascade $39 / 2^{-} \rightarrow 37 / 2^{-} \rightarrow 35 / 2_{2}^{-} \rightarrow 33 / 2^{-} \rightarrow 31 / 2^{-} \rightarrow 29 / 2_{2}^{-}$ $\rightarrow 27 / 2^{-}$. Both the yrast $29 / 2^{-}$and $35 / 2^{-}$states have only very weak feeding and decay branches and do not fit in well with the calculated spectrum (see Fig. 7). We therefore assume that the experimental $29 / 2^{-}$yrast level is a proton state, most probably mainly of $\pi\left(f_{5 / 2}\right)^{-1} \pi\left(g_{9 / 2}\right)^{4}$ configuration since it decays exclusively to the $25 / 2^{-}$core state, whereas the calculated $29 / 2_{1}^{-} \nu p h$ level has a $40 \%$ branch to the core-excited $25 / 2_{2}^{-}$state. The transition strength of the decay from the yrast $29 / 2^{-}$to the yrast $25 / 2^{-}$state is measured to be $B(E 2)>9 e^{2} \mathrm{fm}^{4}$, whereas it is calculated to be $B(E 2)=0.14 e^{2} \mathrm{fm}^{4}$. We assign the second observed $29 / 2_{2}^{-}$ state to the first $29 / 2_{1}^{-}$state in the calculation. As can be read from Table II, this assignment also leads to a much better agreement between theory and experiment for the transition from and into the second observed $29 / 2_{2}^{-}$state. The observed M1 transition rate between the $29 / 2_{2}^{-}$and $29 / 2_{1}^{-}$states can be used to obtain an estimate of the mixing of $\nu p h$ and proton states. If the proton state is $\pi\left(f_{5 / 2}\right)^{-1} \otimes{ }^{94} \mathrm{Ru}\left(12^{+}\right)$, the diagonal matrix element of the $M 1$ operator for this state is calculated to be $44 \mu_{N}$, while the corresponding matrix element for the $\nu p h$ state is $24 \mu_{N}$. The observed $B(M 1)$ of $0.055 \mu_{N}^{2}$ then suggests mixing of just $0.4 \%$. The experimental $35 / 2_{1}^{-}$state most probably contains strong partitions including $f_{5 / 2}$ or $p_{3 / 2}$ protons which are responsible for the fact that the $37 / 2^{-}$yrast state decays to the $35 / 2_{2}^{-}$level and not to the $35 / 2^{-}$yrast state. In this case, however, no further support for this interpretation is obtained from the transition strengths, since no values could be measured for transitions decaying into or from the yrast $35 / 2^{-}$level.

A comparison between the experimental $M 1$ and $E 2$ transition strengths within the main decay sequences of ${ }^{93} \mathrm{Tc}$ and their theoretical counterparts, obtained in the SM1 calculations, is shown in Fig. 8. $M 1$ transitions between $\nu p h$ and core states are forbidden because they would require a $g_{9 / 2}$ $\rightarrow d_{5 / 2}$ transition. Indeed, the observed $M 1$ transition strengths of the $23 / 2^{+} \rightarrow 21 / 2_{1}^{+}$and $27 / 2^{-} \rightarrow 25 / 2_{1}^{-}$transitions are much smaller than the values obtained for M1 transitions among the $\nu p h$ states. For these latter transitions, SM1 predicts strengths of typical $0.1 \mu_{N}^{2}$ and $1 \mu_{N}^{2}$, whereas for the experimental values a larger scattering is observed most 
TABLE II. Experimental reduced electromagnetic transition probabilities in ${ }^{93} \mathrm{Tc}$ deduced in the present work in comparison with the results of the shell model calculations SM1, SM2, and SM3 (see text). The values have been corrected for internal conversion and $\delta=0$ is assumed for all $\Delta I=0,1 M 1$ transitions.

\begin{tabular}{|c|c|c|c|c|c|c|c|c|c|}
\hline \multirow[b]{2}{*}{$E_{x}(\mathrm{keV})$} & \multirow[b]{2}{*}{$I_{i}^{\pi}$} & \multirow[b]{2}{*}{$E_{\gamma}(\mathrm{keV})$} & \multirow[b]{2}{*}{$b(\%)$} & \multirow[b]{2}{*}{$I_{f}^{\pi}$} & \multirow[b]{2}{*}{$\sigma L$} & \multicolumn{4}{|c|}{$B(\sigma L)\left(e^{2} \mathrm{fm}^{2}\right) /\left(e^{2} \mathrm{fm}^{4}\right) /\left(10^{-3} \mu_{N}^{2}\right)$} \\
\hline & & & & & & Expt. & SM1 & $\mathrm{SM} 2$ & SM3 \\
\hline \multicolumn{10}{|c|}{ Positive parity } \\
\hline 2184 & $17 / 2^{+}$ & 750 & 100 & $13 / 2^{+}$ & $E 2$ & $88(16)^{a}$ & 148 & 70.6 & 57.6 \\
\hline 2534 & $21 / 2^{+}$ & 350 & 100 & $17 / 2^{+}$ & $E 2$ & $75(5)$ & 82 & 75.5 & 81.5 \\
\hline \multirow[t]{2}{*}{4257} & $21 / 2_{2}^{+}$ & 330 & $5.7(5)$ & $19 / 2^{+}$ & $M 1$ & $19(3)$ & $210^{b}$ & & \\
\hline & & 1723 & $94.3(3.2)$ & $21 / 2^{+}$ & $M 1$ & $2.2(2)$ & $4.8^{b}$ & 21.2 & 26.5 \\
\hline 4973 & $25 / 2^{+}$ & 2439 & 100 & $21 / 2^{+}$ & $E 2$ & $>7$ & 28 & 139 & 174 \\
\hline \multirow[t]{3}{*}{5076} & $23 / 2^{+}$ & 818 & $74.8(6.9)$ & $21 / 2_{2}^{+}$ & $M 1$ & $>1$ & $380^{\mathrm{b}}$ & 0.68 & 0.509 \\
\hline & & 2541 & $7.2(4.2)$ & $21 / 2^{+}$ & $M 1$ & $>3 \times 10^{-3}$ & 10 & 0.03 & 0.032 \\
\hline & & 1796 & $18.0(6.8)$ & $21 / 2^{-}$ & $E 1$ & $>1.5 \times 10^{-7}$ & & & \\
\hline \multirow[t]{2}{*}{5564} & $25 / 2_{2}^{+}$ & 488 & $35.4(1.5)$ & $23 / 2^{+}$ & $M 1$ & $17(3)$ & 290 & 30.4 & 30.6 \\
\hline & & 1306 & $64.6(2.8)$ & $21 / 2_{2}^{+}$ & $E 2$ & $14(2)$ & $209^{b}$ & 1.24 & 1.04 \\
\hline \multirow[t]{2}{*}{6016} & $27 / 2^{+}$ & 453 & $22.1(1.3)$ & $25 / 2_{2}^{+}$ & $M 1$ & $>1.4$ & 340 & & \\
\hline & & 2129 & $77.9(4.0)$ & $25 / 2^{-}$ & $E 1$ & $>5.3 \times 10^{-7}$ & & & \\
\hline \multirow[t]{2}{*}{6087} & $29 / 2^{+}$ & 70 & $17.9(9)$ & $27 / 2^{+}$ & $M 1$ & $205(22)$ & 550 & 2.45 & 2.82 \\
\hline & & 523 & $82.1(2.0)$ & $25 / 2_{2}^{+}$ & $E 2$ & $118(12)$ & 123 & 3.48 & 2.63 \\
\hline 6669 & $31 / 2^{+}$ & 583 & 100 & $29 / 2^{+}$ & $M 1$ & $846(100)$ & 1880 & & \\
\hline \multirow[t]{2}{*}{7281} & $33 / 2^{+}$ & 612 & $97.4(3.0)$ & $31 / 2^{+}$ & $M 1$ & $>390$ & 1680 & 0.03 & 0.003 \\
\hline & & 1194 & $2.6(1.2)$ & $29 / 2^{+}$ & $E 2$ & $>8$ & 70 & 0.391 & 0.775 \\
\hline 7811 & $35 / 2^{+}$ & 530 & 100 & $33 / 2^{+}$ & $M 1$ & $425(76)$ & 440 & 38.6 & 35.2 \\
\hline 10271 & $39 / 2^{+}$ & 2460 & 100 & $35 / 2^{+}$ & $E 2$ & $>4.7$ & 23 & & \\
\hline \multicolumn{10}{|c|}{ Negative parity } \\
\hline \multirow[t]{2}{*}{3280} & $21 / 2^{-}$ & 746 & $1.7(6)$ & $21 / 2^{+}$ & $E 1$ & $>0.8 \times 10^{-5}$ & & & \\
\hline & & 1096 & $98.3(1.7)$ & $17 / 2^{-}$ & $E 2$ & $>248$ & 216 & 383 & 418 \\
\hline 3887 & $25 / 2^{-}$ & 607 & 100 & $21 / 2^{-}$ & $E 2$ & $115(11)$ & 156 & 198 & 221 \\
\hline 6052 & $25 / 2_{2}^{-}$ & 2165 & 100 & $25 / 2_{1}^{-}$ & $M 1$ & $>7$ & 0.056 & 5.64 & 9.35 \\
\hline \multirow[t]{3}{*}{6372} & $27 / 2^{-}$ & 320 & $6.7(6)$ & $25 / 2_{2}^{-}$ & $M 1$ & $376(41)$ & 310 & & \\
\hline & & 2485 & $88.1(2.8)$ & $25 / 2_{1}^{-}$ & $M 1$ & $11(1)$ & 0.46 & 1.36 & 6.76 \\
\hline & & 1399 & $5.2(8)$ & $25 / 2^{+}$ & $E 1$ & $>3.2 \times 10^{-5}$ & & & \\
\hline 6453 & $29 / 2^{-}$ & 2566 & 100 & $25 / 2^{-}$ & $E 2$ & $>9$ & $(0.14)^{\mathrm{c}}$ & 34.9 & 38.9 \\
\hline \multirow[t]{3}{*}{6856} & $29 / 2_{2}^{-}$ & 484 & $89.4(2.8)$ & $27 / 2^{-}$ & $M 1$ & $340(21)$ & $1200(4.6)^{\mathrm{c}}$ & & 12.8 \\
\hline & & 403 & $8.4(7)$ & $29 / 2^{-}$ & $M 1$ & $55(5)$ & $(19)^{\mathrm{c}}$ & 124 & 78.4 \\
\hline & & 2969 & $2.2(7)$ & $25 / 2^{-}$ & $E 2$ & $0.06(2)$ & $0.14(0.063)^{\mathrm{c}}$ & 171 & 200 \\
\hline \multirow[t]{2}{*}{7372} & $31 / 2^{-}$ & 516 & $73.0(1.9)$ & $29 / 2_{2}^{-}$ & $M 1$ & $235(21)$ & $470(10)^{c}$ & 3.49 & 17.1 \\
\hline & & 1000 & $27.0(1.1)$ & $27 / 2^{-}$ & $E 2$ & 171(16) & 240 & 1.17 & 305 \\
\hline \multirow[t]{3}{*}{7879} & $33 / 2^{-}$ & 507 & $84.0(2.3)$ & $31 / 2^{-}$ & $M 1$ & $>884$ & 1530 & 2.19 & 1370 \\
\hline & & 1023 & $12.6(8)$ & $29 / 2_{2}^{-}$ & $E 2$ & $>213$ & $237^{\mathrm{c}}(1.0)$ & 42.1 & 0.319 \\
\hline & & 1210 & $3.4(1.1)$ & $31 / 2^{+}$ & $E 1$ & $>2 \times 10^{-5}$ & & & \\
\hline 8496 & $35 / 2_{2}^{-}$ & 617 & $91.9(2.7)$ & $33 / 2^{-}$ & $M 1$ & $378(59)$ & $780(160)^{d}$ & 0.322 & 243 \\
\hline & & 1124 & $4.6(9)$ & $31 / 2^{-}$ & $E 2$ & 35(9) & $180(8.0)^{\mathrm{d}}$ & 38.2 & 38.2 \\
\hline & & 1215 & $3.5(1.4)$ & $33 / 2^{+}$ & $E 1$ & $2.1(9) \times 10^{-5}$ & & & \\
\hline 8851 & $37 / 2^{-}$ & 355 & $62.9(1.8)$ & $35 / 2_{2}^{-}$ & $M 1$ & $785(66)$ & $960(350)^{d}$ & 152 & 152 \\
\hline & & 972 & $20.3(1.1)$ & $33 / 2^{-}$ & $E 2$ & $187(18)$ & 148 & 0.223 & 177 \\
\hline & & 1040 & $16.8(9)$ & $35 / 2^{+}$ & $E 1$ & $9.2(9) \times 10^{-5}$ & & & \\
\hline 9419 & $39 / 2^{-}$ & 568 & $79.4(2.3)$ & $37 / 2^{-}$ & $M 1$ & $65(22)$ & 160 & 5.57 & 5.57 \\
\hline & & 923 & $20.6(1.2)$ & $35 / 2_{2}^{-}$ & $E 2$ & $66(23)$ & $131(43)^{d}$ & 18.9 & 18.9 \\
\hline 11525 & $43 / 2^{-}$ & 429 & $15.0(5.2)$ & $41 / 2^{-}$ & $M 1$ & $>18$ & & 331 & 331 \\
\hline & & 2106 & $85.0(11.9)$ & $39 / 2^{-}$ & $E 2$ & $>3.6$ & & 30.3 & 30.3 \\
\hline
\end{tabular}

${ }^{\mathrm{a}}$ Using the literature value $\tau\left(17 / 2^{+}\right)=39(7)$ ps [13].

${ }^{\mathrm{b}}$ Assuming expt. $21 / 2_{2}^{+}$is calc. $21 / 2_{3}^{+}$.

${ }^{c}$ Values in brackets assuming calc. $29 / 2_{1}^{-}$is expt. $29 / 2_{1}^{-}$. The others assuming calc. $29 / 2_{1}^{-}$is expt. $29 / 2_{2}^{-}$.

${ }^{\mathrm{d}}$ Values in brackets assuming calc. $35 / 2_{1}^{-}$is expt. $35 / 2_{1}^{-}$and calc. $35 / 2_{2}^{-}$is expt. $35 / 2_{2}^{-}$. The others assuming calc. $35 / 2_{1}^{-}$is expt. $35 / 2_{2}^{-}$. 


\section{$\pi=+$}



$35 / 2$

7811

7890

$33 / 2^{+}$

7281

7310

$31 / \underline{2}^{+} \quad 6669$

\begin{tabular}{|c|c|c|}
\hline & 6087 & 6190 \\
\hline $12^{+}$ & $\overline{6016}=$ & 6070 \\
\hline $5 / 2^{+}$ & $5564 \quad 25 / 2^{+}$ & 5590 \\
\hline
\end{tabular}

$2 3 / 2 ^ { + } \quad 5 0 7 6 = - - 2 3 \longdiv { 2 ^ { + } } 5 2 3 0$

$25 / 2^{+} \quad 4973--\overline{25 / 2^{+}}$





\begin{tabular}{|c|c|c|}
\hline $11 / 2^{+}$ & $1516 \ldots 11 / 2^{+}$ & 1520 \\
\hline $13 / 2^{+}$ & $13 / 2^{+}$ & 7410 \\
\hline $5 / 2^{+}$ & 1194 & 1250 \\
\hline $7 / 2^{+}$ & $680 \ldots-n / 2^{+}$ & 690 \\
\hline
\end{tabular}

$9 / 2^{+} \quad 0 \quad---\underline{9} / \underline{\underline{2}}^{+} \quad 0$

exp SM1

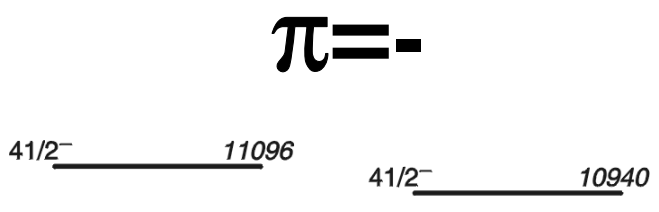

\begin{tabular}{|c|c|c|c|}
\hline & & $37 / 2^{-}$ & 10060 \\
\hline $39 / 2^{-}$ & 9419 & $37 / 2^{-}$ & $\begin{array}{l}9540 \\
\frac{9400}{}\end{array}$ \\
\hline $37 / 2^{-}$ & 9371 & $39 / 2^{-}$ & \\
\hline $37 / 2^{-}$ & 9138 & $37 / 2^{-}$ & 8930 \\
\hline $37 / 2^{-}$ & $\begin{array}{l}8851 \\
8496 \\
\end{array}$ & $\begin{array}{r}35 / 2^{-} \\
35 / 2^{-}\end{array}$ & $\begin{array}{l}8840 \\
8450\end{array}$ \\
\hline
\end{tabular}

33/2- 7879

31/2

29/2- 6856 $--29 / 2^{-} \quad 6860$



$25 / 2^{-}$



21/2- $3280 \ldots-21 / 2^{-} \quad 3260$

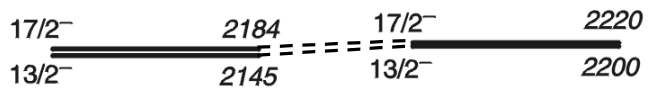

$5 / 2^{-} \quad 1408-\ldots-5 / 2^{-} \quad 1400$

$1 / 2^{-} \quad 392$

exp SM1

FIG. 7. Excitation energies in ${ }^{93} \mathrm{Tc}$ obtained in the shell model calculation SM1 in comparison with the experimental values for the positive parity on the left and the negative parity on the right. 

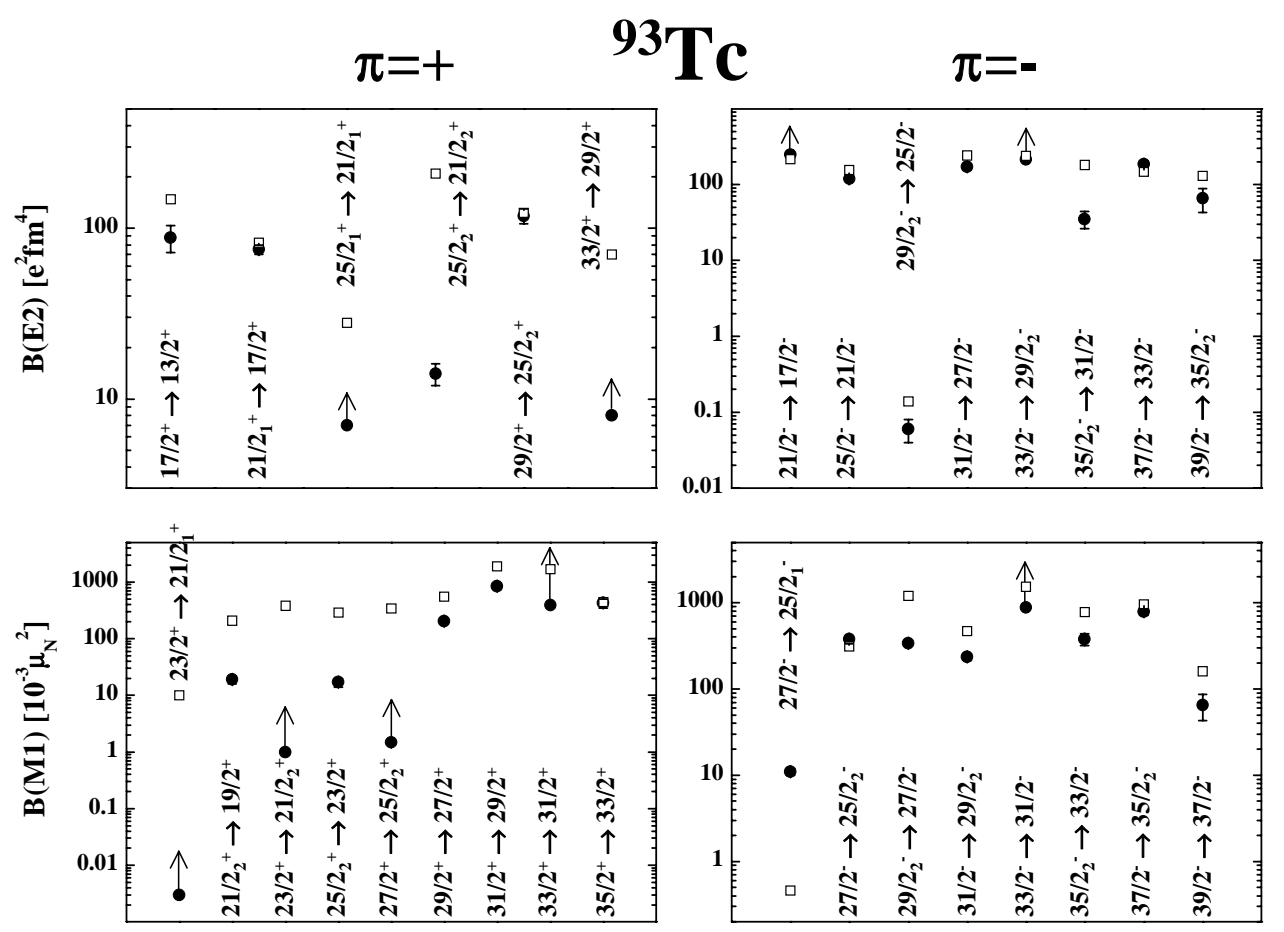

FIG. 8. Comparison between experimental and theoretical (SM1) transition strengths for $M 1$ and $E 2$ transitions in ${ }^{93}$ Tc. The results for the positive parity are shown on the left and the results for the negative parity on the right. The experimental values are marked by filled circles with error bars and labeled by the initial and final spins. The SM1 values resulting from the assignment shown in Fig. 7 are given as open squares.

probably due to admixtures of $f_{5 / 2}, p_{3 / 2}$ proton configurations in the wave functions. $E 2$ transitions between the $\nu p h$ and core states are severely retarded because the $d_{5 / 2} g_{9 / 2}^{-1}$ neutron particle-hole pair is mainly coupled to $J>2$. The $29 / 2_{2}^{-} \rightarrow 25 / 2^{-}$transition is the only one observed of this type and its $E 2$ strengths of $0.06(2) e^{2} \mathrm{fm}^{4}$ indicates indeed a retardation by about three orders of magnitude as compared to the allowed E2 transitions both among the core states as well as among the $\nu p h$ states. In conclusion, the overall features of the electromagnetic transition strengths are well described by the shell model calculation SM1 and together with the good agreement between experimental and calculated level energies, one can conclude that most of the highspin states observed in this nucleus are in fact neutron particle-hole states which include the breakup of the $N=50$ closed shell.

\section{B. Calculations in an extended proton space (SM2, SM3)}

In an attempt to describe simultaneously neutron core excitations and proton excitations from the completely filled $p_{3 / 2}, f_{5 / 2}$ orbits into the $g_{9 / 2}$ subshell, we performed shell model calculations considering a model space consisting of $f_{5 / 2}, p_{3 / 2}, p_{1 / 2}, g_{9 / 2}$ proton and $g_{9 / 2}, d_{5 / 2}$ neutron orbitals relative to a ${ }^{66} \mathrm{Ni}$ core. We had two justifications for not including any further neutron orbits above the $N=50$ gap, e.g., the $g_{7 / 2}$ orbit, which in ${ }^{95} \mathrm{Rh}$ is as close in energy as $0.94 \mathrm{MeV}$ [14] to the $d_{5 / 2}$ orbit. The first is the observation of high-energy $\gamma$ rays $\left(E_{\gamma}>2 \mathrm{MeV}\right)$ above the maximum spin states of the $\pi\left(g_{9 / 2}\right)^{3,4,5} \otimes\left[\nu\left(g_{9 / 2}\right)^{-1} \nu\left(d_{5 / 2}\right)\right] \quad$ and $\left[\pi\left(g_{9 / 2}\right)^{4,5,6} \pi\left(p_{1 / 2}\right)^{-1}\right] \otimes\left[\nu\left(g_{9 / 2}\right)^{-1} \nu\left(d_{5 / 2}\right)\right] \quad$ configurations in all three $N=50$ isotones recently studied. These maximum spin values are $35 / 2^{+}, 39 / 2^{-}$for ${ }^{93} \mathrm{Tc} ; 19^{+}, 20^{-}$in ${ }^{94} \mathrm{Ru}$; and $39 / 2^{+}, 39 / 2^{-}$in ${ }^{95} \mathrm{Rh}$. The observed energy gap above these states indicates that $d_{5 / 2}$ is the only relevant orbit. A second justification comes from the success of describing the level sequences in ${ }^{94} \mathrm{Ru}$ and ${ }^{95} \mathrm{Rh}$ without including further neutron orbits $[1,2]$.

The effective interactions in the proton shells were taken from the work of Ji and Wildenthal [15]. For the protonneutron interaction connecting the $\pi\left(p_{1 / 2}, g_{9 / 2}\right)$ and the $\nu g_{9 / 2}$ orbitals, the two-body matrix elements (TBME) given by Gross and Frenkel [16] were used. Empirical TBME were taken for the $\nu\left(g_{9 / 2}\right) \otimes \nu\left(d_{5 / 2}\right)[17]$ and $\pi\left(f_{5 / 2}\right) \otimes \nu\left(g_{9 / 2}\right)$ [18] multiplets, while the surface delta interaction was used for the remaining TBME [19]. The single-particle energies of the proton orbitals relative to the ${ }^{66} \mathrm{Ni}$ core were taken to reproduce those relative to ${ }^{78} \mathrm{Ni}$ [15]. Similarly, the neutron single-particle energies were adopted to fit the neutron single-hole energy of the $g_{9 / 2}$ orbital [16] and the neutron single-particle energy of the $d_{5 / 2}$ shell [20], relative to a ${ }^{88} \mathrm{Sr}$ core. More detailed information about all parameters used can be found in the paper by Winter et al. [19]. The only restriction we applied in our calculations, which were performed using the computer code RITSSCHIL [21], is that the excitation of only one neutron from the $g_{9 / 2}$ orbital over the shell gap into the $d_{5 / 2}$ orbital was allowed. In the following, this calculation will be referred to as SM2. The level energies 

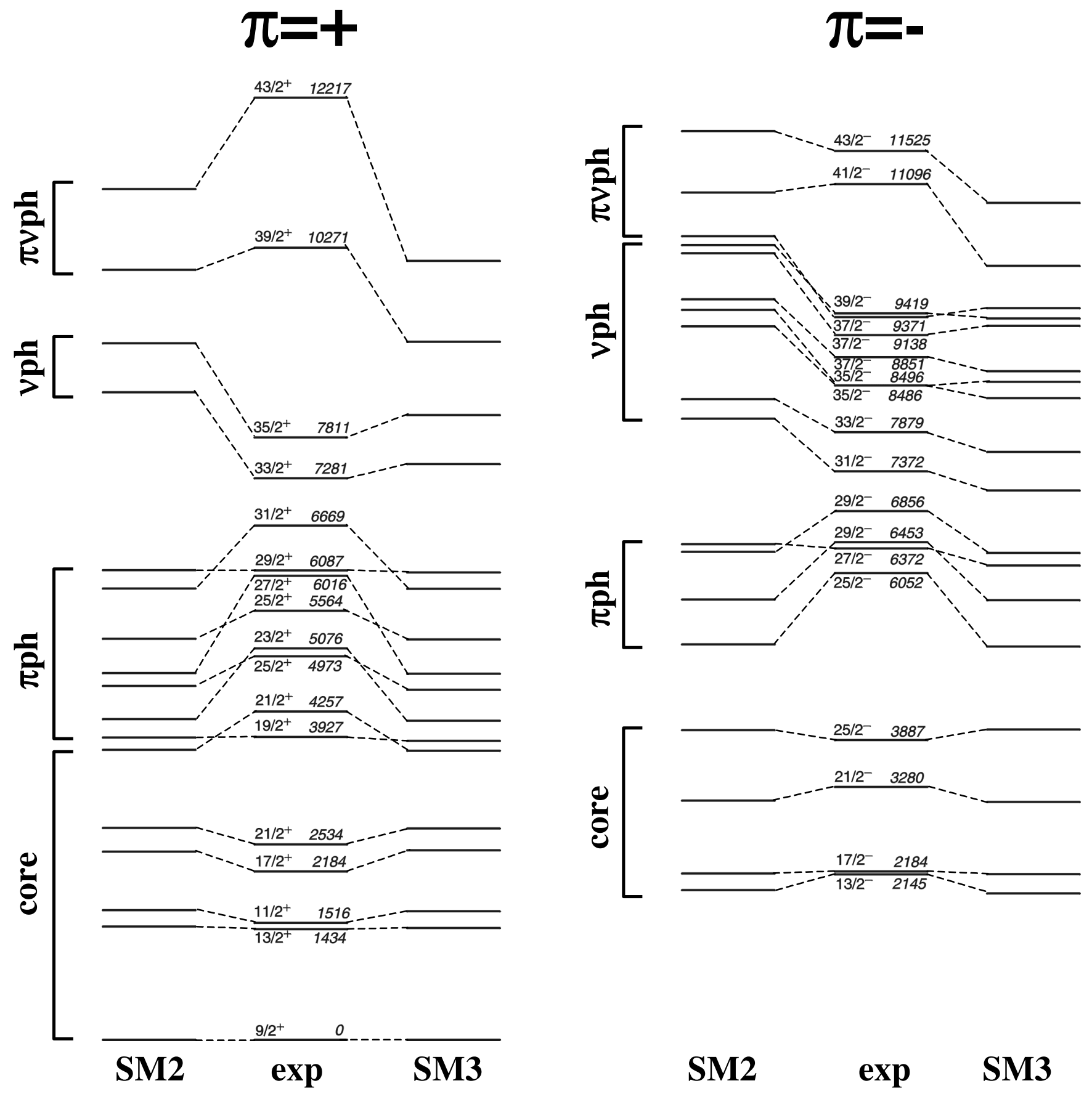

FIG. 9. Excitation energies in ${ }^{93}$ Tc obtained in the shell model calculations SM2 and SM3 in comparison with the experimental values for the positive parity on the left and the negative parity on the right.

obtained in SM2 are compared to the experimental values in Fig. 9. The states are grouped into core, $\pi p h, \nu p h$, and $\pi \nu p h$ states according to the main component of their wave functions (except for the $33 / 2^{-}$state, which we will discuss later). Two observations are evident at first sight. First, all yrast states in the spin range $19 / 2^{+} \leqslant 31 / 2^{+}$, except for the $21 / 2^{+}$level at positive parity as well as the negative parity $27 / 2^{-}$and $29 / 2^{-}$yrast states, have main components including a $f_{5 / 2} \rightarrow g_{9 / 2}$ proton excitation in the SM2 calculation. This means that up to the highest possible spin values, which can be obtained in the $\left(f_{5 / 2}, p_{3 / 2}, p_{1 / 2}, g_{9 / 2}\right)$ configuration space without breaking the $N=50$ neutron shell closure, namely $31 / 2^{+}$and $29 / 2^{-}$, the proton configurations $\pi\left(f_{5 / 2}^{-1} p_{1 / 2}^{-1} g_{9 / 2}^{5}\right)$ and $\pi\left(f_{5 / 2}^{-1} g_{9 / 2}^{4}\right)$, respectively, are energetically favored as compared to the neutron core-excited configurations $\pi\left(g_{9 / 2}^{3}\right) \nu\left(g_{9 / 2}^{-1} d_{5 / 2}^{1}\right)$ and $\pi\left(p_{1 / 2}^{-1} g_{9 / 2}^{4}\right) \nu\left(g_{9 / 2}^{-1} d_{5 / 2}^{1}\right)$, respectively. The second observation is that all neutron coreexcited states ( $\nu p h$ states) are shifted to higher excitation energies as compared to the experiment and also relative to states of lower spin containing no core excitation. The same effect had already been found in earlier shell model studies of ${ }^{86} \mathrm{Kr}$ [19] and ${ }^{89} \mathrm{Y}$ [22] using the same parameter set and 
also in our recent studies of the two $N=50$ isotones ${ }^{94} \mathrm{Ru}$ [2] and ${ }^{95} \mathrm{Rh}[1]$.

As in our work on ${ }^{94} \mathrm{Ru}$ and ${ }^{95} \mathrm{Rh}$, we therefore repeated the calculation with a modified $d_{5 / 2}$ neutron single-particle energy, which we reduced by $1 \mathrm{MeV}$. We will refer to this calculation in the following by SM3. Note that all parameters used in SM3 are identical to the ones used not only to describe the excitation energies, but furthermore to successfully reproduce the electromagnetic transition strengths in ${ }^{94} \mathrm{Ru}$ and ${ }^{95} \mathrm{Rh}[1,2]$. The calculated excitation energies are compared to both the experiment and the SM2 calculation in Fig. 9. As can be read from this figure, the reduction of the $d_{5 / 2}$ neutron single-particle energy had very little influence on the states characterized by proton excitations but lowered the excitation energies of the core-excited states. However, and most important, it did not change the order of the states, still the proton-excited states are yrast up to the highest-spin values possible in the $\left(f_{5 / 2}, p_{3 / 2}, p_{1 / 2}, g_{9 / 2}\right)$ space.

We already know from our discussion of the SM1 shell model calculation in the preceding section that the measured transition strengths between the states above 3.9 and $6 \mathrm{MeV}$ at positive and negative parity are better reproduced by neutron core-excited configurations. Exceptions are the yrast $29 / 2^{-}$state, which seems to be a proton-excited level, and the $35 / 2_{1}^{-}$and $37 / 2_{2}^{-}$states, which are of mixed $\pi \nu p h$ structure. Since the SM3 calculation does not reproduce these structures it is not surprising that the calculated transition strengths, which are listed in Table II, do not agree very well with the experimental values. Drastic examples are the $33 / 2^{+} \rightarrow 31 / 2^{+}$and the $33 / 2^{-} \rightarrow 29 / 2_{2}^{-}$transitions for which calculated and experimental values differ by five and three orders of magnitude, respectively. One reason may be that whereas in the calculation these are forbidden transitions between $\nu p h$ and $\pi p h$ states, the rather large experimental values suggest initial and final states to belong to the same family of states, namely, the neutron core-excited states.

The unsatisfactory description of high-spin states in SM2, SM3 is caused by the fact that in this model space proton excitations from the $p f$ shell seem to be favored as compared to neutron core excitations. At positive parity, for example, the proton-excited states with the configuration $\pi\left(f_{5 / 2}^{-1} p_{1 / 2}^{-1} g_{9 / 2}^{5}\right)$ are yrast up to the highest possible spin of $31 / 2^{+}$. Above this maximum spin value the states, e.g., $33 / 2^{+}$and $35 / 3^{+}$, have in SM2 and SM3 the same main component $\pi\left(g_{9 / 2}^{3}\right) \nu\left(g_{9 / 2}^{-1} d_{5 / 2}^{1}\right)$ as in SM1, however, with a smaller partition and additional admixtures of the type $\pi\left(f_{5 / 2}^{-1} p_{1 / 2}^{-1} g_{9 / 2}^{5}\right) \nu\left(g_{9 / 2}^{-1} d_{5 / 2}^{1}\right) \quad$ which obviously interfere destructively. To further study this problem, we performed additional calculations using the same parameters as used in SM2 and SM3, but excluding proton excitations from the $f p$ shell to the $g_{9 / 2}$ orbital. In these calculations, the level ordering as well as the transition strengths are very close to the results of the SM1 calculation. This leads to the conclusion that the parameters in the SM2 and SM3 calculations, which are responsible for favoring proton excitations over neutron core excitations, are not adequate for a correct description of the interplay between these two competing excitation modes in the nucleus ${ }^{93} \mathrm{Tc}$. Note that this weak point of the SM2 and SM3 calculations did not appear in our study of the isotone ${ }^{95} \mathrm{Rh}$ [1] because in that case, with five protons in the $g_{9 / 2}$ orbit, the gain in spin for proton excitations is much lower than in ${ }^{93} \mathrm{Tc}$ and with it the spin range in which both excitation modes compete.

\section{ANALOGIES BETWEEN THE $N=50$ ISOTONES ${ }^{93} \mathrm{Tc}$, ${ }^{94} \mathbf{R u}$, AND ${ }^{95} \mathbf{R h}$}

The lowest negative parity states in the isotones ${ }^{93} \mathrm{Tc}$ and ${ }^{95} \mathrm{Rh}$ are built from a $p_{1 / 2}$ proton coupled to four $g_{9 / 2}$ protons in the case of Tc and six $g_{9 / 2}$ protons or four $g_{9 / 2}$ proton holes in Rh. Taking into account the particle-hole symmetry of the independent particle picture, both situations are expected to be identical. Furthermore, the positive parity states in the even nucleus ${ }^{94} \mathrm{Ru}$ also contain four active $g_{9 / 2}$ protons. Assuming that the $p_{1 / 2}$ proton in the $\pi=-$ states of ${ }^{93} \mathrm{Tc}$ and ${ }^{95} \mathrm{Rh}$ acts only as a spectator, it is expected that corresponding states (states with the same $g_{9 / 2}$ proton configuration) differing by $1 / 2 \hbar$ in spin should have the same structure in all three isotones and consequently the transitions between these states comparable strengths. This expectation is further supported by the facts that $p_{1 / 2}$ does not contribute to the $E 2$ matrix element and that the $M 1$ transitions are dominated by the large magnetic moment of the $g_{9 / 2}$ protons. The $E 2$ and $M 1$ transition probabilities for the main sequences in ${ }^{93} \mathrm{Tc}$, ${ }^{94} \mathrm{Ru}[2]$, and ${ }^{95} \mathrm{Rh}[1]$ are shown in Fig. 10. Here, $I_{i}^{+} \rightarrow I_{f}^{+}$ transitions in ${ }^{94} \mathrm{Ru}$ are compared to the corresponding $\left(I_{i}\right.$ $+1 / 2)^{-} \rightarrow\left(I_{f}+1 / 2\right)^{-}$transitions in ${ }^{93} \mathrm{Tc}$ and ${ }^{95} \mathrm{Rh}$. This comparison shows that the $p_{1 / 2}$ proton can indeed be regarded as a spectator, since the similarity between the experimental and calculated strengths in all three isotones is striking. For the electric quadrupole transitions, strengths around $B(E 2) \approx 100 e^{2} \mathrm{fm}^{4}$ are observed for the $18^{+} \rightarrow 16^{+} \rightarrow 14^{+}$, $12^{+} \rightarrow 10^{+} \rightarrow 8^{+}, \quad 37 / 2^{-} \rightarrow 33 / 2^{-} \rightarrow 29 / 2^{-}, \quad$ and $25 / 2^{-}$ $\rightarrow 21 / 2^{-} \rightarrow 17 / 2^{-}$sequences. These large strengths are mainly due to allowed recouplings of the $\pi\left(g_{9 / 2}\right)^{n}$ parts of the wave functions with $\Delta I_{\pi}=2$. The forbidden $14^{+} \rightarrow 12^{+}$ and $29 / 2^{-} \rightarrow 25 / 2^{-}$transitions from neutron core-excited to pure proton states, on the other hand, are retarded by about two orders of magnitude. A similar observation is made for the magnetic dipole transitions. Whereas rather strong $M 1$ transitions in the range $0.1 \mu_{N}^{2}-1 \mu_{N}^{2}$ are observed in all three nuclei which have there origin in allowed recouplings of the two unpaired neutrons (the $g_{9 / 2}$ neutron hole and the $d_{5 / 2}$ neutron) to different spin values while keeping the proton part of the wave function constant, the $13^{+} \rightarrow 12^{+}$and $27 / 2^{-} \rightarrow 25 / 2^{-}$transitions are retarded because they require a forbidden $d_{5 / 2} \rightarrow g_{9 / 2}$ neutron transition.

\section{CONCLUSIONS}

Accurate lifetimes in ${ }^{93} \mathrm{Tc}$ obtained from a high-statistics $\gamma \gamma$ coincidence RDDS experiment via the reaction ${ }^{64} \mathrm{Zn}\left({ }^{35} \mathrm{Cl}, \alpha 2 p\right)$ have been presented. The experimental high-spin states and electromagnetic decay properties of ${ }^{93} \mathrm{Tc}$ were compared to different shell model calculations based on 

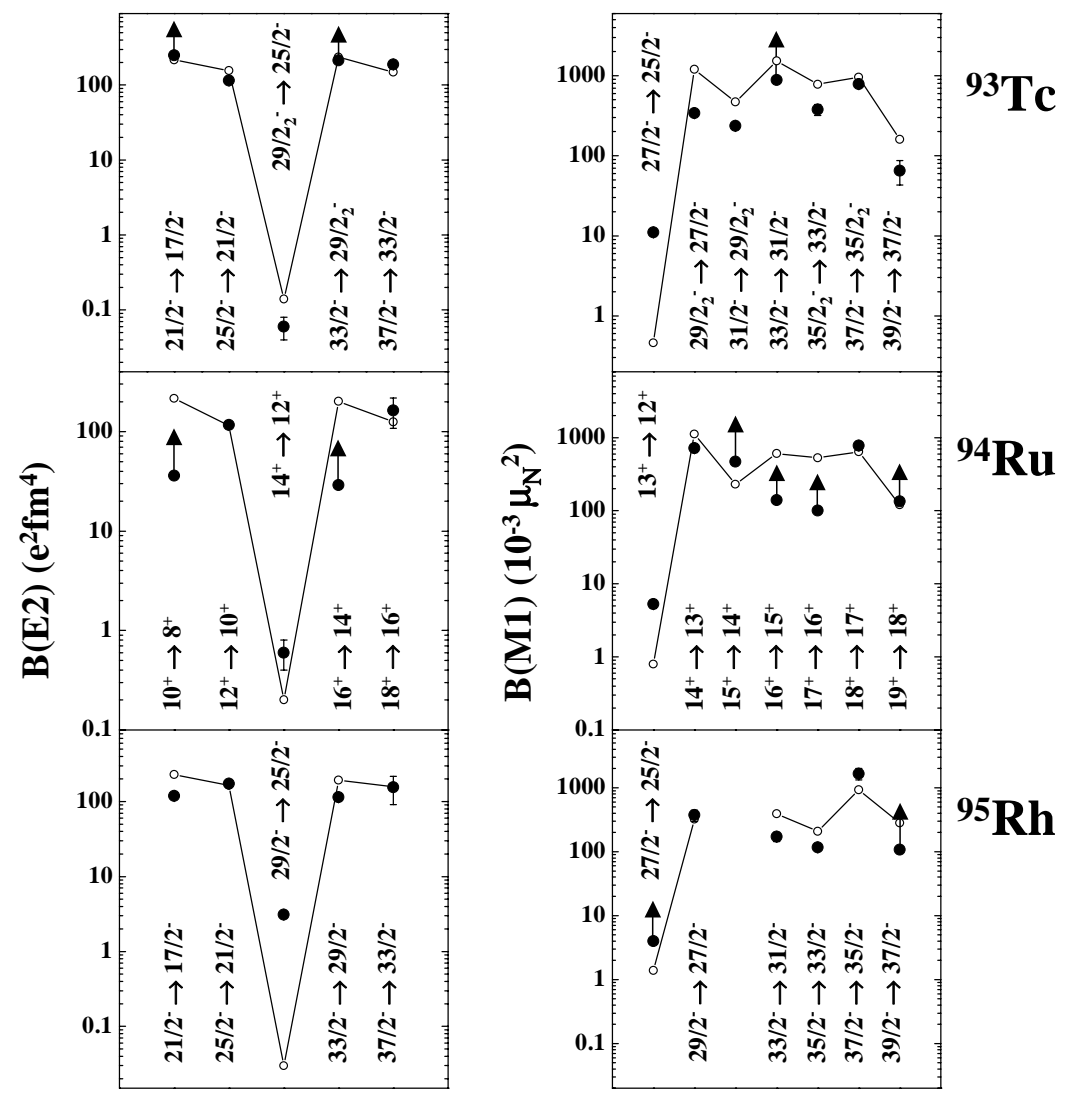

FIG. 10. Comparison between transition strengths at negative parity in ${ }^{93} \mathrm{Tc}$ and ${ }^{95} \mathrm{Rh}$ and at positive parity in ${ }^{94} \mathrm{Ru}$. The $E 2$ transition strengths are shown on the left and the $M 1$ strengths on the right. The experimental values are marked by filled circles with error bars and labeled by the initial and final spins. The SM1 values are given as open circles connected by full lines.

extended configuration spaces, including both one-particleone-hole excitations across the $N=50$ neutron shell as well as proton excitations from the completely filled $p_{3 / 2}$ and $f_{5 / 2}$ orbits into the $g_{9 / 2}$ subshell. The $29 / 2^{-}$yrast state has been identified by means of the decay strengths to be an intruder state involving an $f_{5 / 2}$ proton hole, but no neutron excitation. All other levels above $4 \mathrm{MeV}$ at positive parity and $6 \mathrm{MeV}$ at negative parity with the exception of the yrast $25 / 2^{+}$state, which has a stretched $g_{9 / 2}^{5}$ proton configuration, contain one neutron being excited from the $g_{9 / 2}$ into the $d_{5 / 2}$ orbit.

\section{ACKNOWLEDGMENTS}

We are most grateful to the crew of the XTU tandem accelerator at the LNL Legnaro for their friendly and efficient cooperation. This work has been supported by the European Commission through the Contract No. HPRI-1999CT-00083 and the Deutsches Bundesministerium für Bildung, Wissenschaft, Forschung und Technologie (BMBF). A. Jungclaus acknowledges financial support from the Deutsche Forschungsgemeinschaft (DFG) within the Heisenberg program.
[1] A. Jungclaus, D. Kast, K.P. Lieb, C. Teich, M. Weiszflog, T. Härtlein, C. Ender, F. Köck, D. Schwalm, J. Reif, R. Peusquens, A. Dewald, J. Eberth, H.-G. Thomas, M. Górska, and H. Grawe, Nucl. Phys. A637, 346 (1998).

[2] A. Jungclaus, D. Kast, K.P. Lieb, C. Teich, M. Weiszflog, T. Härtlein, C. Ender, F. Köck, D. Schwalm, I.P. Johnstone, J. Reif, R. Schwengner, R. Peusquens, A. Dewald, J. Eberth, H.-G. Thomas, M. Górska, and H. Grawe, Phys. Rev. C 60, 014309 (1999).

[3] M. Grecescu, A. Nilsson, and L. Harms-Ringdahl, Nucl. Phys. A212, 429 (1973).

[4] S.S. Ghugre, S.B. Patel, M. Gupta, R.K. Bhowmik, and J.A. Sheikh, Phys. Rev. C 47, 87 (1993).

[5] H.A. Roth, S.E. Arnell, D. Foltescu, O. Skeppstedt, J. Blomqvist, A. Nilsson, T. Kuroyanagi, S. Mitarai, and J. Nyberg, Phys. Rev. C 50, 1330 (1994).
[6] I.P. Johnstone and L.D. Skouras, Phys. Rev. C 55, 1227 (1997).

[7] A. Dewald, P. Sala, R. Wrzal, G. Böhm, D. Lieberz, G. Siems, R. Wirowski, K.O. Zell, A. Gelberg, P. von Brentano, P. Nolan, A.J. Kirwan, J. Bishop, R. Julin, A. Lampinen, and H. Hattula, Nucl. Phys. A545, 822 (1992).

[8] E. Galindo et al., Phys. Rev. C (submitted).

[9] C. Rossi Alvarez, Nucl. Phys. News 3, 10 (1993).

[10] G. Böhm, A. Dewald, P. Petkov, and P. von Brentano, Nucl. Instrum. Methods Phys. Res. A 329, 248 (1993); A. Dewald, S. Harissopulos, and P. von Brentano, Z. Phys. A 334, 163 (1989).

[11] W.D. Schneider, K.H. Gonsior, and C. Günther, Nucl. Phys. A249, 103 (1975).

[12] B.A. Brown, D.B. Fossan, P.M.S. Lesser, and A.R. Poletti, Phys. Rev. C 13, 1194 (1976). 
[13] C. Broude et al., Z. Phys. A 336, 133 (1990).

[14] Table of Isotopes, 8th ed., edited by R.B. Firestone et al. (Wiley, New York, 1996).

[15] X. Ji and B.H. Wildenthal, Phys. Rev. C 37, 1256 (1988).

[16] R. Gross and A. Frenkel, Nucl. Phys. A267, 85 (1976).

[17] P.C. Li and W.W. Daehnick, Nucl. Phys. A462, 26 (1987).

[18] P.C. Li, W.W. Daehnick, S.K. Saha, J.D. Brown, and R.T. Kouzes, Nucl. Phys. A469, 393 (1987).
[19] G. Winter, R. Schwengner, J. Reif, H. Prade, L. Funke, R. Wirowski, N. Nicolay, A. Dewald, P. von Brentano, H. Grawe, and R. Schubart, Phys. Rev. C 48, 1010 (1993).

[20] K. Muto, T. Shimano, and H. Horie, Phys. Lett. 135B, 349 (1984).

[21] D. Zwarts, Comput. Phys. Commun. 38, 365 (1985).

[22] J. Reif, G. Winter, R. Schwengner, H. Prade, and L. Käubler, Nucl. Phys. A587, 449 (1995). 